\title{
Once-weekly teriparatide administration for 24 weeks in postmenopausal women with osteoporosis: reply to $\mathrm{T}$. Kawada
}

\author{
T. Sugimoto • T. Nakamura • Y. Nakamura • Y. Isogai • \\ M. Shiraki
}

Received: 11 April 2014 / Accepted: 15 April 2014 / Published online: 8 May 2014

(C) The Author(s) 2014. This article is published with open access at Springerlink.com

\section{Dear Editor,}

We thank Dr. Kawada for both his interest in our current study [1] and his important comment [2]. As Dr. Kawada pointed out, our study did not directly compare changes in bone metabolic markers between once-weekly and daily administration of teriparatide. Therefore, we cited a reference in the "Discussion" section about the differences in the changes of these markers between groups [1]. This point was also mentioned as a study limitation in the last paragraph of the "Discussion." As Dr. Kawada also pointed out, the differences in the changes in bone metabolic markers between once-

\footnotetext{
T. Sugimoto

Internal Medicine 1, Faculty of Medicine, Shimane University, 89-1

Enya-cho, Izumo, Shimane 693-8501, Japan

T. Nakamura

National Center for Global Health and Medicine, 1-21-1 Toyama, Shinjuku-ku, Tokyo 162-8655, Japan

e-mail: t-nak@utopia.ocn.ne.jp

Y. Nakamura $\cdot$ Y. Isogai

Project for Bone Metabolic Disease, Asahi Kasei Pharma

Corporation, 1-105 Kanda Jinbocho, Chiyoda-ku, Tokyo 101-8101, Japan

Y. Nakamura

e-mail: nakamura.ycp@om.asahi-kasei.co.jp

Y. Isogai

e-mail: isogai.yb@om.asahi-kasei.co.jp

M. Shiraki

Research Institute and Practice for Involutional Diseases, 1610-1

Meisei, Misato, Azumino, Nagano 399-8101, Japan

e-mail: ripid@fc4.so-net.ne.jp

T. Sugimoto $(\bowtie)$

Department of Internal Medicine 1, Shimane University Faculty of Medicine, 89-1 Enya-cho, Izumo, Shimane 693-8501, Japan

e-mail: sugimoto@med.shimane-u.ac.jp
}

weekly and daily administrations may be partially affected by the conditions under which samples are collected, such as the timing of sampling. Regarding this point, we cited another reference about serial changes in bone metabolic markers up to 2 weeks after a single subcutaneous $56.5 \mu \mathrm{g}$ dose of teriparatide [3].

In incident vertebral fractures, relative risk reductions (RRR) of 65 and $80 \%$ have been reported with daily and onceweekly administrations, respectively [4, 5]. However, these vertebral fractures were evaluated by different methods. When similar evaluation methods were used, a similar fracture reduction rate between daily administration overseas and onceweekly administration in Japan has also been reported (RRR of $84 \%$ with daily administration) [6].

With regard to his comment about ethnic differences, Tsujimoto et al. investigated whether any differences could be found in pharmacokinetics (AUC and Cmax) and effects of daily administration on BMD between Japanese and nonJapanese patients [7]. They subsequently reported no differences in PK parameters or BMD changes between Japanese and Caucasian patients. In addition, characteristics of Japanese patients did not differ markedly between our current study of once-weekly administration and another study of daily administration $[1,8]$. Moreover, patients' characteristics did not differ markedly between studies of daily administration in Japanese and non-Japanese patients [4, 8]. Therefore, ethnic differences and patients' characteristics did not play a substantial role in any differences in changes in bone metabolic markers between once-weekly and daily administration. Our study patients comprised postmenopausal osteoporotic women who had some features typical of postmenopausal Japanese women.

Values with a non-normal distribution like bone metabolic markers are often analyzed by natural log transformation or non-parametric methods. A matched-pair signed-rank (Wilcoxon) test and natural log-transformed analysis 
confirmed statistically significant differences that were similar to our report.

Finally, the main purpose of our study was to investigate whether there was any loss of responsiveness of bone metabolic markers up to 24 weeks. As Dr. Kawada pointed out, no comparison was made with controls; however, no decrease was observed in responsiveness after 24 weeks [1]. In addition, based on the results of changes in bone metabolic markers in the TOWER trial with once-weekly administration up to 72 weeks [5], this responsiveness may be expected to persist for up to 72 weeks.

Conflicts of interest TS has received research grants and consulting fees from the pharmaceutical companies Asahi Kasei Pharma and Daiichi Sankyo. TN has received research grants and/or consulting fees from the pharmaceutical companies Chugai, Teijin, Asahi Kasei Pharma, and Daiichi Sankyo. YN and YI are employees of Asahi Kasei Pharma Corporation. MS has received consulting fees from the pharmaceutical companies Asahi Kasei Pharma, Dai-ichi Sankyo, Chugai, and Teijin Pharma.

Open Access This article is distributed under the terms of the Creative Commons Attribution Noncommercial License which permits any noncommercial use, distribution, and reproduction in any medium, provided the original author(s) and the source are credited.

\section{References}

1. Sugimoto T, Nakamura T, Nakamura Y, Isogai Y, Shiraki M (2014) Profile of changes in bone turnover markers during once-weekly teriparatide administration for 24 weeks in postmenopausal women with osteoporosis. Osteoporos Int 25:1173-1180

2. Kawada T (2014) Once-weekly teriparatide administration for 24 weeks in postmenopausal women with osteoporosis. Osteoporos Int. doi:10.1007/s00198-014-2721-6

3. Shiraki M, Sugimoto T, Nakamura T (2013) Effects of a single injection of teriparatide on bone turnover markers in postmenopausal women. Osteoporos Int 24:219-226

4. Neer RM, Arnaud CD, Zanchetta JR, Prince R, Gaich GA, Reginster JY, Hodsman AB, Eriksen EF, Ish-Shalom S, Genant HK, Wang O, Mitlak BH (2001) Effect of parathyroid hormone (1-34) on fractures and bone mineral density in postmenopausal women with osteoporosis. N Engl J Med 344:1434-1441

5. Nakamura T, Sugimoto T, Nakano T, Kishimoto H, Ito M, Fukunaga M, Hagino H, Sone T, Yoshikawa H, Nishizawa Y, Fujita T, Shiraki M (2012) Randomized teriparatide [human parathyroid hormone (PTH) 1-34] Once-Weekly Efficacy Research (TOWER) trial for examining the reduction in new vertebral fractures in subjects with primary osteoporosis and high fracture risk. J Clin Endocrinol Metab 97: 3097-3106

6. Prevrhal S, Krege JH, Chen P, Genant H, Black DM (2009) Teriparatide vertebral fracture risk reduction determined by quantitative and qualitative radiographic assessment. Curr Med Res Opin 25: 921-928

7. Tsujimoto M, Uenaka K, Iwata A, Higashiuchi Y, Sowa H (2012) Effects of teriparatide in Japanese and non-Japanese populations: bridging findings on pharmacokinetics and efficacy. J Bone Miner Metab 30:326-337

8. Miyauchi A, Matsumoto T, Sugimoto T, Tsujimoto M, Warner MR, Nakamura T (2010) Effects of teriparatide on bone mineral density and bone turnover markers in Japanese subjects with osteoporosis at high risk of fracture in a 24-month clinical study: 12-month, randomized, placebo-controlled, double-blind and 12-month open-label phases. Bone 47:493-502 